\title{
Alteration in cardiac PI3K/Akt/mTOR and ERK signaling pathways with the use of growth hormone and swimming, and the roles of miR21 and miR133
}

\author{
ORKIDE PALABIYIK ${ }^{1}$, EBRU TASTEKIN ${ }^{2}$, ZEYNEP BANU DOGANLAR $^{3}$, \\ PINAR TAYFUR $^{4}$, AYTEN DOGAN $^{3}$ and SELMA ARZU VARDAR ${ }^{4}$
}

\begin{abstract}
${ }^{1}$ Department of Medical Services and Techniques, Trakya University Health Services Vocational College; Departments of ${ }^{2}$ Pathology, ${ }^{3}$ Medical Biology and ${ }^{4}$ Physiology, Trakya University Faculty of Medicine, Edirne 22030, Turkey
\end{abstract}

Received September 10, 2018; Accepted December 5, 2018

DOI: $10.3892 /$ br.2018.1179

\begin{abstract}
Athletes misuse recombinant human growth hormone $(\mathrm{r}-\mathrm{hGH})$ to enhance their performance. Although $\mathrm{r}-\mathrm{hGH}$ is known to increase cardiac hypertrophy, the underlying molecular mechanism remains unclear. The aim of the present study was to investigate the role of $\mathrm{r}-\mathrm{hGH}$ in cardiac intracellular signaling pathways and of miR-21 and miR-133 expression in rat hearts during exercise. A total of 36 adult male Sprague-Dawley rats were divided into sedentary control $(\mathrm{SC}, \mathrm{n}=9)$, swimming exercise (SE, $\mathrm{n}=8), \mathrm{r}-\mathrm{hGH}(\mathrm{GH}, \mathrm{n}=10)$ and swimming exercise plus $\mathrm{r}-\mathrm{hGH}(\mathrm{SE}-\mathrm{GH}, \mathrm{n}=9)$ groups. The exercise groups completed a 1-h swimming exercise 5 times a week for 8 weeks. Subcutaneous r-hGH was administered as $0.3 \mathrm{mg} / \mathrm{kg} / \mathrm{day}$. Phosphoinositide-3-kinase (PI3K), serine/threonine protein kinase 1 (AKT1), extracellular signal-regulated kinase (ERK), microRNA (miR)-21 and miR-133 expression was evaluated in ventricular muscle by real-time quantitative polymerase chain reaction. Protein expression of PI3K, AKT1, ERK and mechanistic target of rapamycin (mTOR) was also assessed by immunohistochemistry. Statistical differences were analyzed by two-way ANOVA. PI3K and AKT1 expression and the gene and protein levels was notably increased in the SE-GH group compared with in SC ventricular tissues $(\mathrm{P}<0.05)$. mTOR protein expression was higher in the GH, SE and SE-GH groups compared with in the SC group $(\mathrm{P}<0.05$, $<0.05$ and $<0.001$, respectively). ERK gene/protein expression was similar across all groups. miR-21 and miR-133 levels in ventricular muscle were higher in the SE and GH groups than those in the SC group. In summary, growth hormone application coupled with swimming exercise appeared to affect the
\end{abstract}

Correspondence to: Dr Orkide Palabiyik, Department of Medical Services and Techniques, Trakya University Health Services Vocational College, Balkan Campus, Edirne 22030, Turkey

E-mail: orkide_69@hotmail.com

Key words: growth hormone, microRNA, heart, swimming, cardiac hypertrophy
PI3K/AKT/mTOR signaling pathway in the left ventricular tissue of rats; however, ERK signaling pathway appeared inactive in physiological left ventricular hypertrophy caused by swimming and GH administration over 8 weeks. Furthermore, GH treatment resulted in increased miR-21 and miR-133 expression. Future study by our group will aim to assess the effects of higher dose GH treatment.

\section{Introduction}

Growth hormone (GH), a 191-amino acid single-chain peptide, is a peptide hormone with anabolic and performance-enhancing effects (1). Direct effects of GH include increasing muscle mass (2) Most of the indirect effects are mediated by induction of insulin-like growth factor 1 (IGF-1) expression in the liver and in peripheral tissues (3). An organ most affected by GH imbalance (deficiency or excess) is the heart (4). Bodybuilders and athletes misuse recombinant human $\mathrm{GH}(\mathrm{r}-\mathrm{hGH})$ preparations to increase muscle mass and strength; as such, r-hGH has been on the prohibited substances list of the International Federations and the International Olympic Committee since 1989. It is also on the 2010 World Anti-Doping Agency list of prohibited substances $(5,6)$.

Left ventricular hypertrophy resulting from swimming exercise is an important physiological mechanism that increases sarcomeres and cardiac cell growth to compensate for the chronic increase in hemodynamic load (7). Conversely, pathological left ventricular hypertrophy caused by cardiovascular disease is associated with increased fibrosis and decreased aerobic capacity leading to incompatible remodeling in cardiac cells (7). Both in physiological and pathological hypertrophy, intermediate signal transduction pathways in the cytoplasm are triggered via membrane-bound receptors. Signal transduction pathways critical for myosin growth include phosphoinositide-3-kinase (PI3K)/serine/threonine protein kinase (AKT)/mechanistic target of rapamycin (mTOR), mitogen-activated protein kinase (MAPK)/extracellular signal-regulated kinase (ERK), protein kinase $\mathrm{C}$ and nuclear factor of activated $\mathrm{T}$ cells pathways, among others (8). In particular, the PI3K/AKT/mTOR signaling pathway has been reported to serve a regulatory role in cell growth, metabolism, 
survival and angiogenesis, and to govern the development and transformation of left ventricular hypertrophy $(7,9)$. The ERK signaling pathway, the members of which belong to the MAPK family, comprises a cascade consisting of a series of successively acting kinases that finally results in the phosphorylation and activation of terminal kinases including p38, c-Jun N-terminal kinases and ERK. Following activation, each ERK phosphorylates intracellular targets including transcription factors, resulting in reprogramming of cardiac gene expression $(10,11)$.

MicroRNAs (miRNA/miRs) are small RNA molecules of 18-25 nucleotides which do not encode proteins, and negatively regulate gene expression $(12,13)$. They are involved in the regulation of several physiological responses including cell apoptosis (14), proliferation and differentiation (15). In addition, recent studies have demonstrated that certain miRNAs are involved in cardiovascular diseases and pathological left ventricular hypertrophy (16-18). In particular, miR-21 and miR-133 have been demonstrated to regulate cardiac hypertrophy $(17,19)$. miR-21 has also been implicated in the regulation of cardiac fibrosis and cardiac muscle contractility (20), and has been shown to negatively regulate phosphatase and tensin homolog (PTEN) gene expression (7).

It is established that r-hGH may cause cardiac hypertrophy (6). However, to the best of our knowledge, there is no study demonstrating the mechanism through which r-hGH, together with swimming exercise, affects signaling pathways (PI3K/AKT/mTOR and ERK) that regulate cardiac signaling genes and the miRNAs (miR-21 and miR-133) targeting the genes in these signaling pathways. Thus, the present study aimed to investigate i) the effect of r-hGH and exercise on cardiac PI3K/AKT/mTOR and ERK signaling pathways, and ii) the role of miR-21 and miR-133 expression in ventricular muscle.

\section{Materials and methods}

Animals and exercise protocols. A total of 36 male Sprague-Dawley rats (14 weeks old) weighing 200-250 g were obtained from the Experimental Animals Center of Trakya University, Edirne, Turkey. All animals were fed daily with tap water and a standard pellet diet for rats under optimal laboratory conditions (temperature: $22 \pm 2^{\circ} \mathrm{C}$; humidity: $50-55 \%$; light/dark cycle: $12 / 12 \mathrm{~h}$ ). The animals were randomly stratified into sedentary control ( $\mathrm{SC}, \mathrm{n}=9$ ), swimming exercise ( $\mathrm{SE}$, $\mathrm{n}=8), \mathrm{r}-\mathrm{hGH}(\mathrm{GH}, \mathrm{n}=10)$ and swimming exercise plus $\mathrm{r}-\mathrm{hGH}$ (SE-GH, n=9) groups. For 8 weeks, from Monday to Friday, the rats in the SE and SE-GH groups completed a swimming exercise for $1 \mathrm{~h}$ per day. A low-intensity, long-period swimming exercise protocol was applied $(7,21)$. The exercise protocol was selected as it has been tested in previous studies and been determined to be effective in demonstrating cardiovascular adaptations and muscular oxidative capacity $(21,22)$. Animals in the swimming exercise groups were allowed to swim for $1 \mathrm{~h}$ wearing a weight of $5 \%$ of their body weight attached to the tail. Prior to the exercise program, the rats in the swimming exercise groups were subjected to an adaptation period of 5 days during which the duration and load were gradually increased according to increase in animal weight. Animals that could not adapt to the experiment (refused to swim or prone to plunging) were excluded from the study. In addition, animals in the non-exercise groups ( $\mathrm{SC}$ and $\mathrm{GH}$ ) were exposed to pool water by means of an apparatus placed inside the pool for 8 weeks, and from Monday to Friday, the rats in the SE group completed a 1-h swimming exercise (7). The swimming pool $(120 \times 80 \times 75 \mathrm{~cm})$ was filled with water to a depth of $65 \mathrm{~cm}$. The pool was divided by plastic barriers into twelve lanes. To avoid interaction, each rat was placed into an individual lane. Water temperature was maintained at $31 \pm 1^{\circ} \mathrm{C}$.

r-hGH was administered subcutaneously 5 days per week for 8 weeks to the animals in the GH and SE-GH groups at a dose of 0.3 (1 IU/day) $\mathrm{mg} / \mathrm{kg}$ (Humatrope ${ }^{\circledR}$, Eli Lilly and Company, Indianapolis, IN, USA). The dose was selected based on previous studies (18-21). Animals in the SE-GH group were injected subcutaneously immediately prior to exercise. Saline at the same dose $(0.3 \mathrm{mg} / \mathrm{kg})$ was injected subcutaneously to the animals in the SE group.

The study was conducted in the Trakya University Local Experimental Animals Research Center after obtaining approval from the institutional Experimental Animals Ethics Committee (approval no. TUHADYEK:2015/10).

Preparation of samples. After fasting for $24 \mathrm{~h}$ following the last exercise, the rats received intraperitoneal thiopental $(100 \mathrm{mg} / \mathrm{kg})$ for anesthesia. All rats were sacrificed by cervical dislocation and blood $(5 \mathrm{ml})$ was obtained from the heart for metabolic investigations. Serum was separated from the blood samples via centrifugation at 2,600 $\mathrm{x}$ g for $10 \mathrm{~min}$ at room temperature, and stored at $-20^{\circ} \mathrm{C}$ to measure IGF-1 and insulin levels at a later time point. Measurement of IGF1 and insulin was performed by means of ELISAs. Isolated hearts were weighed and portions of the left ventricular tissue were stored at $-80^{\circ} \mathrm{C}$ for mRNA and miRNA measurements, and the remaining left ventricular tissue was utilized to create paraffin blocks for immunohistochemical evaluation.

Real-time reverse transcription-quantitative polymerase chain reaction $(R T-q P C R)$ analyses

Total RNA isolation and real-time PCR for $m R N A$. Total RNA was isolated from the stored left ventricular tissues using a Total RNA PureLink ${ }^{\circledR}$ RNA Mini kit as per the manufacturer's instructions (Invitrogen; Thermo Fisher Scientific, Inc., Waltham, MA, USA). The quantity of extracted RNA was determined with a Qubit ${ }^{\circledR}$ Fluorometer (Invitrogen; Thermo Fisher Scientific, Inc.).

RNA concentrations of the samples were adjusted to $200 \mathrm{ng} / \mu \mathrm{l}$ and cDNA synthesis was performed using a High Capacity cDNA Reverse Transcription kit (Applied Biosystems; Thermo Fisher Scientific, Inc.). Gene expression was analyzed by means of RT-qPCR. The PCR cycling conditions were an initial denaturation at $95^{\circ} \mathrm{C}$ for $5 \mathrm{~min}$ followed by 40 cycles at $95^{\circ} \mathrm{C}$ for $15 \mathrm{sec}$ and $60^{\circ} \mathrm{C}$ for $45 \mathrm{sec}$ and $72^{\circ} \mathrm{C}$ for $15 \mathrm{sec}$. The primers, sequences of which are provided in Table I, were used with SYBR ${ }^{\circledR}$ Select Master Mix (Applied Biosystems; Thermo Fisher Scientific, Inc.) to determine the changes in gene expression. Gene expressions were determined as the relative fold change compared with the control group, and normalized to $\beta$-actin mRNA expression.

TaqMan miRNA assay and real-time PCR for miRNA. miRNA isolation from left ventricular tissues was performed 
Table I. Quantitative real-time polymerase chain reaction primers.

\begin{tabular}{ll}
\hline Gene & \multicolumn{1}{c}{ Sequence, 5'-3' $^{\prime}$} \\
\hline PI3K & F: CATGGATGCTTTGCAGGGTTT \\
& R: CCAGATGTTCTCCATGATTCGGA \\
PTEN & F: AGACCATAACCCACCACAGC \\
& R: TACACCAGTCCGTCCTTTCC \\
AKT1 & F: ACTGACATTGGACGGCTGAG \\
& R: CAGGTGGGACTGTGATACGG \\
ERK1/2 & F: GAGCCCAGGGGAACTGCT \\
& R: TGGAAGCGGGCTGTCTC \\
ANP & F: CTTCGGGGGTAGGATTGAC \\
& R: CTTGGGATCTTTTGCGATCT \\
F-MHC & R: CATCCCCAATGAGACGAAG \\
& F: AGGCTCTTTCTGCTGGACA \\
& R: AGGAAGGAAGGCTGGAAGAGA
\end{tabular}

PI3K, phosphoinositide-3-kinase; PTEN, phosphatase and tensin homolog; AKT1, serine/threonine protein kinase 1; ERK1/2, extracellular signal-regulated kinase; ANP, atrial natriuretic polypeptide; MHC, myosin heavy chain; F, forward; R, reverse.

with a mirVana miRNA Isolation kit (Ambion) as per the manufacturer's instructions. cDNA for miRNA analysis was synthesized from total RNA utilizing gene-specific primers according to the TaqMan MicroRNA Reverse Transcription Assay protocol (Applied Biosystems; Thermo Fisher Scientific, Inc.). The $15 \mu \mathrm{l}$ reactions obtained by the TaqMan MicroRNA Reverse Transcription kit protocol were incubated in a thermal cycler $\left(10 \mathrm{~min}\right.$ at $95^{\circ} \mathrm{C}$ followed by 40 cycles of $15 \mathrm{sec}$ at $95^{\circ} \mathrm{C}$, $1 \mathrm{~min}$ at $60^{\circ} \mathrm{C}$ and $15 \mathrm{sec}$ at $72^{\circ} \mathrm{C}$ ). PCR quantification was performed by means of a TaqMan MicroRNA Assay (Thermo Fisher Scientific, Inc.) protocol. The probe mix from the TaqMan MicroRNA Assay protocol contained sequences for miR-21 and -133 (Taqman MicroRNA Assay 002393 and 002246; Thermo Fisher Scientific, Inc.). Samples were normalized by evaluating against U6 gene expression (TaqMan MicroRNA Assay 001973).

Quantification. PCR product generation was monitored by measuring the increase in fluorescence caused by probe presence in the TaqMan MicroRNA Assay at each annealing phase or by the binding of SYBR-Green to double-stranded DNA. A melting curve in the SYBR-Green analysis was generated at the end of the reaction to determine that only one product was amplified. Each assay was performed in duplicate. The relative quantities of target gene expression between sedentary rats and rats subjected to swimming exercise with and without $\mathrm{r}-\mathrm{hGH}$ treatment were compared following normalization to the values of internal control $(\Delta \mathrm{Cq})$. Fold change in mRNA and miRNA expressions were calculated using the differences in $\Delta \mathrm{Cq}$ values between two samples $(\Delta \Delta \mathrm{Cq})$ and the equation, $2^{-\Delta \Delta \mathrm{Cq}}(23)$. The results were expressed as a percentage of the sedentary control group.
ELISA measurements. The stored serum samples were evaluated using ELISA kits for IGF-1 (Rat IGF-1, cat no. EK0377; Wuhan Boster Biological Technology, Ltd., Wuhan, China) and insulin (Rat/Mouse insulin, cat no. EZRMI-13K; EMD Millipore, Billerica, MA, USA) as per the manufacturer's instructions.

In the experimental procedure for IGF-1 measurement, a standard sandwich technique involving enzyme-linked immunosorbent technology was used. Absorbance was measured at $450 \mathrm{~nm}$ with an ELISA reader (FL 800; BioTek Instruments, Inc., Winooski, VT, USA). The IGF-1 assay sensitivity was $<5 \mathrm{pg} / \mathrm{ml}$ and the range of the assay was $62.5-4.000 \mathrm{pg} / \mathrm{ml}$.

In the experimental procedure for insulin measurement, a double-antibody sandwich enzyme-linked immunosorbent assay was used to determine insulin level in the samples. Absorbance was measured at $450 \mathrm{~nm}$ with the ELISA reader. The insulin assay sensitivity was $0.2 \mathrm{ng} / \mathrm{ml}(17.5 \mathrm{pM}$ for a sample volume of $10 \mu \mathrm{l}$ ) and the appropriate range of the assay was $0.2-10 \mathrm{ng} / \mathrm{ml}$.

Two repeats were performed.

Immunohistochemical method and evaluation. Left ventricular tissue samples were fixed for $24 \mathrm{~h}$ at room temperature in $10 \%$ formalin solution. The samples were subsequently passed through an ascending ethyl alcohol series (60.0-70.0-80.0-90.0-99.9\%). The tissues were exposed to two changes of xylene for transparency and then embedded in paraffin. Following deparaffinization of $5-\mu \mathrm{m}$ sections from the blocks, one section of each block was stained with hematoxylin and eosin (H\&E) stain for general histological examination of the tissues. Counterstaining was performed with Hematoxylin for 3-5 min at room temperature and eosin for different time periods under visual control. For the other sections, a Ventana BenchMark XT Automated IHC/ISH slide staining system (Ventana Medical Systems, Inc., Tucson, AZ, USA) was used to apply immunohistological stains against the following: PI3K catalytic subunit $\alpha$ (polypeptide p110; PIK3CA; cat no. 09-482), PTEN (cat. no. 09-035), AKT1 [phosphorylated (p)-AKT Ser473], cat no. 07-1398), p-mTOR (p-mTOR Ser2448 catalytic domain, cat no. 07-1415; all from EMD Millipore) and ERK1/2 (p-ERK1/2 Thr202/Thr204; cat no. 4370S; Cell Signaling Technology, Inc., Danvers, MA, USA).

Immunohistochemical scoring was conducted in a manner entirely blinded to all clinical and biological variables (24). PIK3CA staining was scored on the following scale: 0 , no staining; $1+$, staining $<50 \% ; 2+$, staining $\geq 50 \%$ with weak intensity; $3+, \geq 50 \%$ staining with strong intensity (25).

PTEN expression was scored independently based on the intensity and extent of staining. The intensity of positive staining was scored from 0 to 2 as follows: 0 (none), 1 (weak; intensity < positive control), 2 (strong; intensity $\geq$ positive control). Positive staining was assessed using a semi-quantitative grading system consisting of five categories: $0,<5 \%$ positive cells; $1,6-25 \%$ positive cells; $2,26-50 \%$ positive cells; $3,51-75 \%$ positive cells; $4,76-100 \%$ positive cells. The sum of the two values provided the total score, and a score $<4$ was considered PTEN-negative (26).

p-AKT, p-mTOR and ERK levels were stratified into three categories based on staining intensity as well as posi- 
Table II. Effects of r-hGH and exercise on physiological properties and metabolic parameters in rats.

$$
\text { P-value }{ }^{\mathrm{a}}
$$

\begin{tabular}{|c|c|c|c|c|c|c|c|}
\hline Parameters & $\mathrm{SC}(\mathrm{n}=10)$ & $\mathrm{SE}(\mathrm{n}=8)$ & GH $(n=10)$ & SE-GH (n=9) & SE effect & GH effect & $\mathrm{GH}+\mathrm{SE}$ effect \\
\hline $\mathrm{BW}, \mathrm{g}$ & $307.80 \pm 6.34$ & $269.38 \pm 7.09$ & $281.50 \pm 6.34$ & $275.11 \pm 6.69$ & $<0.05$ & 0.13 & $<0.05$ \\
\hline HWI & $3.64 \pm 0.03$ & $4.21 \pm 0.07$ & $4.02 \pm 0.06$ & $4.40 \pm 0.07$ & $<0.05$ & 0.08 & $<0.001$ \\
\hline LVWT, mm & $3.09 \pm 0.06$ & $3.13 \pm 0.06$ & $3.14 \pm 0.05$ & $3.23 \pm 0.08$ & 0.23 & 0.67 & 0.29 \\
\hline Insulin, mIU/l & $0.68 \pm 0.13$ & $0.44 \pm 0.08$ & $0.58 \pm 0.12$ & $0.57 \pm 0.11$ & 0.89 & 0.29 & 0.34 \\
\hline IGF-1,pg/ml & $5,736.05 \pm 306.62$ & $6,410.46 \pm 226.46$ & $6,260.12 \pm 202.55$ & $5,922.10 \pm 142.87$ & 0.69 & 0.97 & 0.23 \\
\hline
\end{tabular}

Data are presented as the mean \pm standard error of the mean. ${ }^{a}$ Compared with SC. BW, body weight; HWI, heart weight index (heart weight/ body weight x1,000 g/g); LVWT, left ventricular wall thickness; IGF-1, insulin-like growth hormone-1; SC, sedentary control; SE, swimming exercise; GH, r-hGH exposure; SE-GH, swimming exercise-r-hGH exposure; r-hGH, recombinant human growth hormone.

tive frequency according to a previously described scoring method (9) with slight modification. Tissues where $<10 \%$ of the cells exhibited weak staining were scored as 0 , tissues where $>10 \%$ of the cells exhibited weak staining or $<20 \%$ exhibited strong staining were scored as 1 , and tissues where $>20 \%$ of the cells exhibited strong staining were scored as 2 (26).

Statistical analysis. All data are presented as the mean \pm standard error of the mean. All statistical analyses were conducted using SPSS for Windows v21.0 (IBM Corp., Armonk, NY, USA). GraphPad Prism for Windows v6.03 (GraphPad Software, Inc., La Jolla, CA, USA) was used to construct the graphs. The one-sample Kolmogorov Smirnov test was used to evaluate the normal distribution of data. The effects of $\mathrm{GH}$ administration and swimming exercise were analyzed by two-way analysis of variance. The Bonferroni post-hoc test was applied for post-hoc comparisons between groups, and $\mathrm{P}<0.05$ was considered to indicate a statistically significant difference.

\section{Results}

Clinical characteristics. Results of all physiological and metabolic parameters of the study evaluating the effects of r-hGH administration and swimming exercise are presented in Table II. Comparison of heart weights revealed an increase in the SE-GH group compared with in the SC group $(\mathrm{P}<0.05)$. This significant difference was deemed to result from the swimming exercise and $\mathrm{r}-\mathrm{hGH}$ administration. In terms of left ventricular weight, this was significantly increased in the SE-GH group $(\mathrm{P}<0.05)$ compared with in the SC group (data not shown). Cardiac hypertrophy grade was determined by heart weight/body weight ratio, referred to as the heart weight index (HWI; heart weight / body weight x1,000 g/g) $(27,28)$. Significant increase in this ratio was observed in the SE $(\mathrm{P}<0.05)$ and $\mathrm{SE}-\mathrm{GH}(\mathrm{P}<0.001)$ groups compared with in the $\mathrm{SC}$ group. The difference in left ventricular wall thickness, as another parameter used to determine cardiac hypertrophy was not statistically significant between the groups (28). Further, no significant differences were observed between the groups when $\mathrm{r}$-hGH administration plus exercise was evaluated for the effect on the two metabolic parameters, insulin and IGF-1.
Molecular markers of left ventricular hypertrophy. Atrial natriuretic peptide (ANP), the two forms of myosin heavy chain ( $\alpha$-MHC and $\beta$-MHC) and $\alpha$-actin, which are considered as molecular markers of cardiac hypertrophy (29), were evaluated. There was a significant increase in ANP expression in the SE-GH group compared with in the SC group (3.20-fold, $\mathrm{P}<0.05)$. $\beta$-MHC was increased in the SE-GH group (6.76-fold, $\mathrm{P}<0.05)$ and decreased in the SE group (0.34-fold, $\mathrm{P}<0.05)$ compared with in the control group (Fig. $1 ; \alpha-\mathrm{MHC}$ and $\alpha$-actin not shown).

Expression analysis of genes in the PI3K/AKT/mTOR and ERK signaling pathways

$P C R$ results. PI3K gene expression, which is established to serve an essential role in regulating cardiac growth (7) was significantly higher in the $\mathrm{SE}-\mathrm{GH}$ group $(5.98$-fold, $\mathrm{P}<0.05)$ compared with in the SC group. Significant increases were also observed in the SE and GH groups (4.30- and 3.67-fold, $\mathrm{P}<0.05)$. PTEN is a major negative regulator of PI3K gene expression (7). The present data demonstrated that PTEN was significantly decreased in the $\mathrm{SE}$ group $(0.44$-fold, $\mathrm{P}<0.05)$ compared with in the SC group; while no significant difference was indicated when the two GH-treated groups were compared with the SC group (Fig. 2).

AKT-1 gene expression, which is involved in the regulation of protein synthesis and is a major suppressor of PI3K (7), was significantly increased in the SE-GH group (3.35-fold, $\mathrm{P}<0.05$ ) compared with in the SC group. The SE and GH groups were similar to the SC group in terms of AKT-1 expression (Fig. 2).

Gene expression of ERK1/2 was unchanged with exercise and/or $\mathrm{r}$-hGH administration and remained comparable across all groups (Fig. 2).

Immunohistochemical results. PI3K $\alpha$ staining grades were significantly different across the groups. The difference was a result of significantly increased PI3K staining in left ventricular tissue of the SE, GH and SE-GH groups compared with that in the SC group (Fig. 3). PTEN staining grades were similar across all groups (not shown).

In terms of AKT, the groups revealed statistically significant differences, with increased p-AKT-1 in the GH and SE-GH groups compared with in the SC group $(\mathrm{P}<0.05)$. Regarding p-mTOR, significant increases were observed in all 

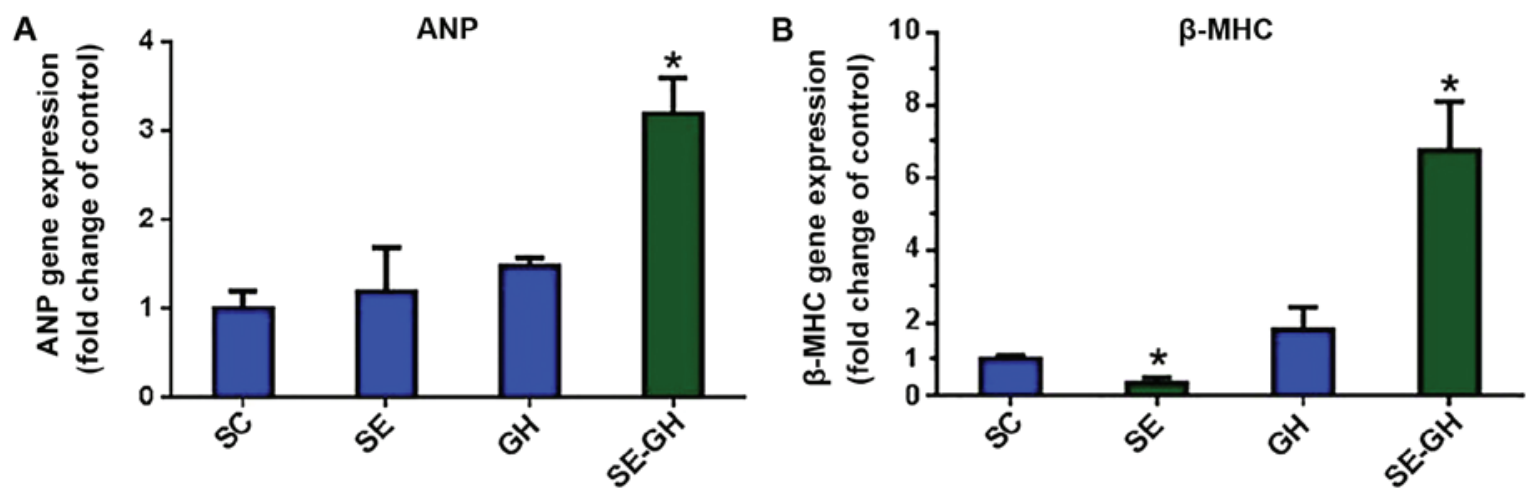

Figure 1. Effect of swimming exercise and/or r-hGH administration on molecular markers of pathological left ventricular hypertrophy. Data are presented as the mean \pm standard error of the mean. (A) ANP; (B) $\beta$-MHC. ${ }^{*} \mathrm{P}<0.05$. ANP, atrial natriuretic peptide; $\beta$-MHC, $\beta$-myosin heavy chain; SC, sedentary control; $\mathrm{SE}$, swimming exercise; GH, r-hGH exposure; SE-GH, swimming exercise-r-hGH exposure; r-hGH, recombinant human growth hormone.
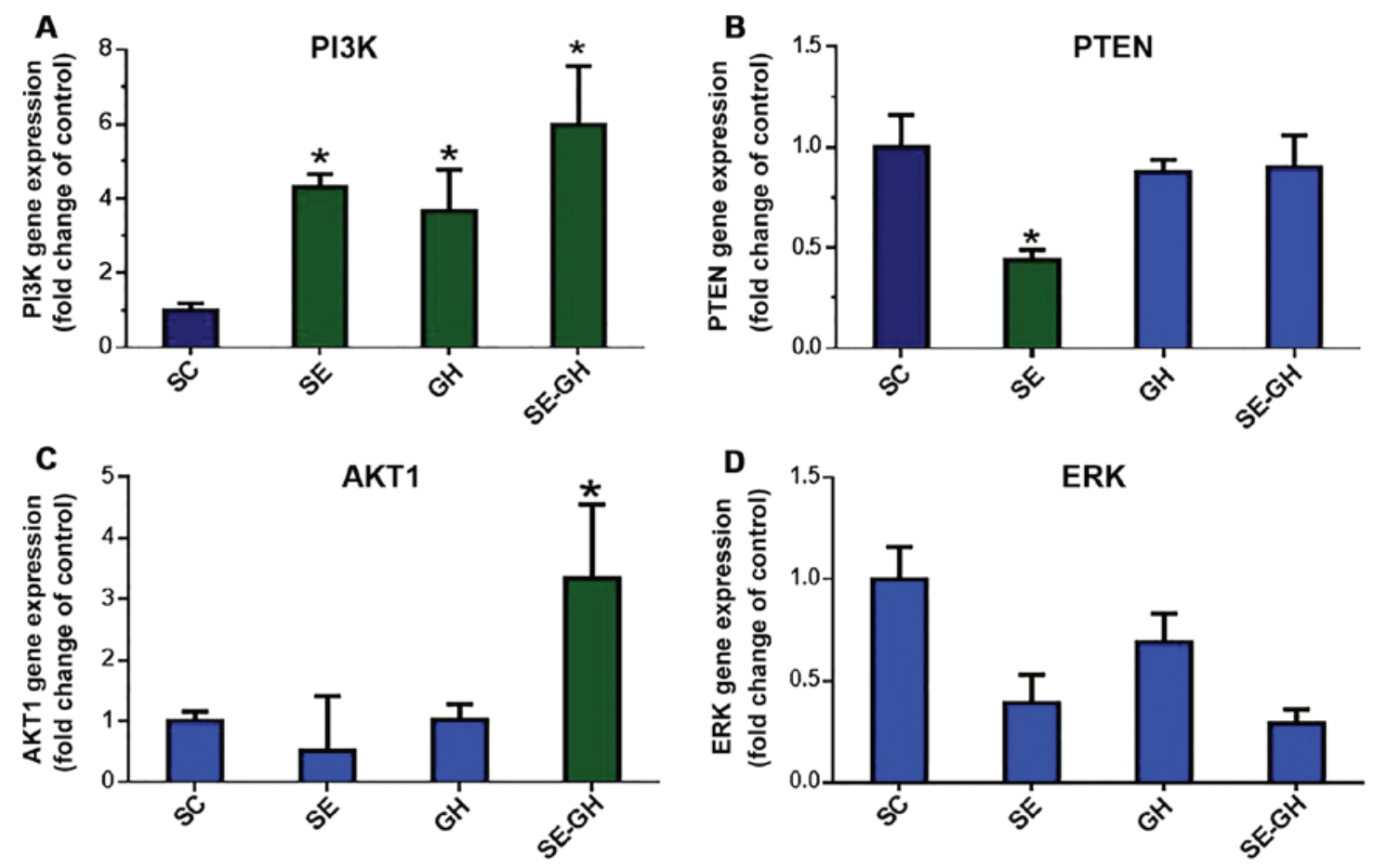

Figure 2. Effect of swimming exercise and/or r-hGH administration on PI3K/AKT/mechanistic target of rapamycin and ERK1/2 signaling pathways in the left ventricular tissue. (A) PI3K gene expression; (B) PTEN gene expression; (C) AKT1 gene expression; (D) ERK gene expression. Data are presented as the mean \pm standard error of the mean. ${ }^{\text {P }}<0.05$ vs. SC group. PI3K, phosphoinositide-3-kinase; PTEN, phosphatase and tensin homolog; ERK, extracellular signal-regulated kinase; SC, sedentary control; SE, swimming exercise; GH, r-hGH exposure; SE-GH, swimming exercise-r-hGH exposure; r-hGH, recombinant human growth hormone.

test groups $[\mathrm{SE}(\mathrm{P}<0.05), \mathrm{GH}(\mathrm{P}<0.05)$ and $\mathrm{SE}-\mathrm{GH}(\mathrm{P}<0.01)]$ compared with SC (Fig. 3).

No significant differences were noted in ERK1/2 protein levels across the groups (not shown).

miRNA analysis. miR-21 gene expression was determined to be increased in the SE and GH groups, compared with the $\mathrm{SC}$ group $(\mathrm{P}<0.05)$. When miR-133 levels were compared, significant increases were observed in the SE and GH groups relative to levels in the $\mathrm{SC}$ group $(\mathrm{P}<0.05)$. Expression levels of miR-21/133 in the SE-GH group did not exhibit any significant change compared with those in the SC group (Fig. 4).

\section{Discussion}

It is known to use r-hGH to improve performance and muscle mass by some athletes (30). However, no study to our knowledge has demonstrated the mechanism through which $\mathrm{r}$-hGH, together with swimming exercise, may affect signaling pathways (PI3K/AKT/mTOR and ERK) that regulate cardiac signaling genes and associated miRNAs (miR-21 and miR-133) (31). A key finding of the present study was the altered expression of genes in the PI3K/AKT/mTOR signaling pathway with $\mathrm{r}-\mathrm{hGH}$ administration and swimming exercise. With this, the present study demonstrated 
A

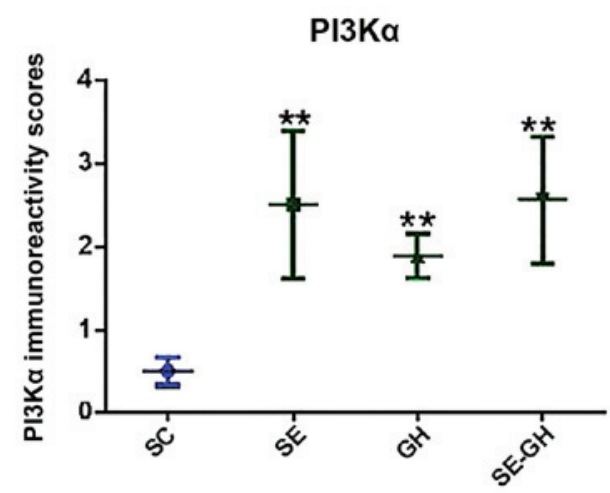

B

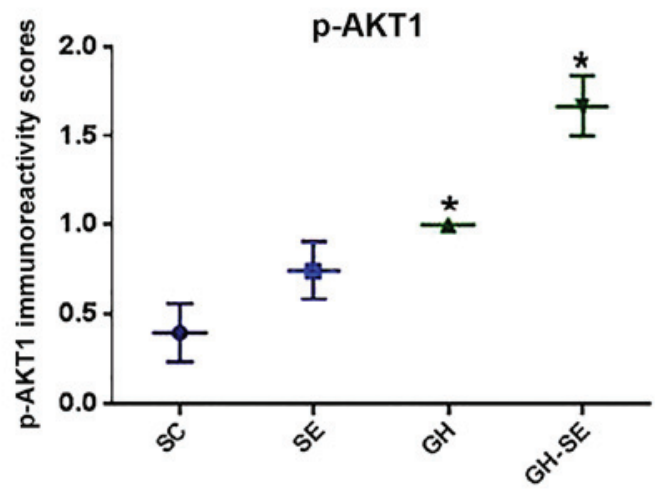

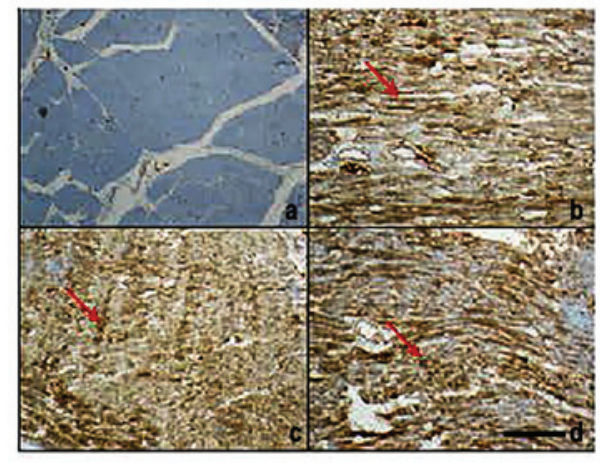
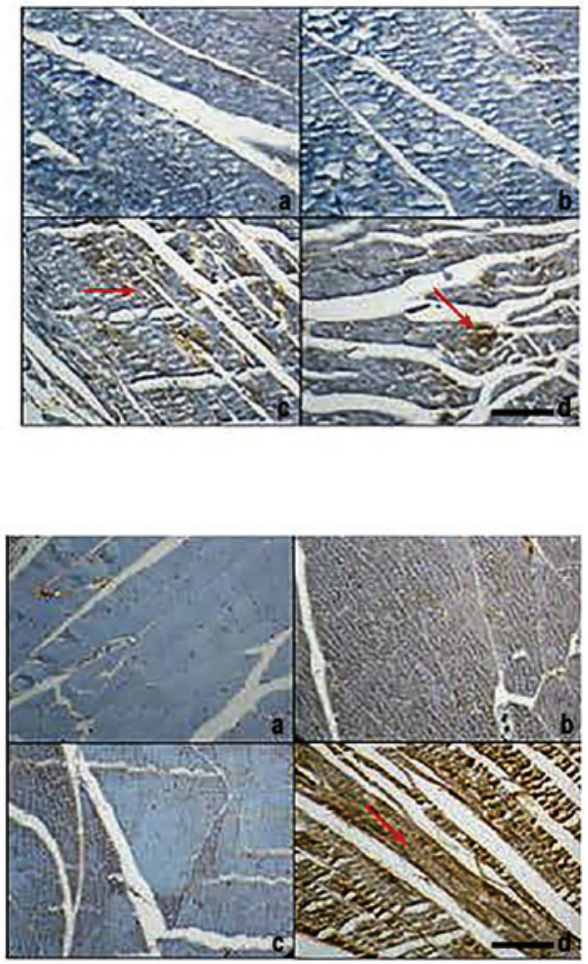

Figure 3. Immunoperoxidase assay of the PI3K/AKT/mTOR signaling pathway. (A) Cardiac P13K $\alpha$ antibody. (A-a) No staining was detected in the SC group. (A-b) Diffuse strong positive staining was detected in the SE, (A-c) GH and (A-d) SE-GH groups. (B) p-AKT antibody. (B-a) No staining was detected in the SC and (B-b) SE groups. (B-c) Weak staining was detected in the (B-d) SE-GH group. (C) p-mTOR antibody. (C-a) No staining was detected in the SC group. (C-b) Diffuse-moderate staining was detected in the SE group. (C-c) Focally moderate staining was detected in the GH group and (C-d) diffuse-strong staining was detected in the SE-GH group. Red arrows indicate areas where staining was apparent. Original magnification, $\mathrm{x} 200$, scale bar, $100 \mu \mathrm{m}$. ${ }^{*} \mathrm{P}<0.05,{ }^{* * *} \mathrm{P}<0.001$. PI3K $\alpha$, phosphoinositide-3-kinase catalytic $\alpha$ (polypeptide p-110); p-AKT, phospho-AKT (Ser473); p-mTOR, phospho-mechanistic target of rapamycin (Ser2448-catalytic domain); SC, sedentary control; SE, swimming exercise; GH, r-hGH exposure; SE-GH, swimming exercise-r-hGH exposure; r-hGH, recombinant human growth hormone.

that r-hGH administration may trigger the similar signal transduction pathways in ventricular muscle. However, no change was detected between the groups in terms of ERK1/2 gene expression, which is involved in the ERK signaling pathway that is active during cardiac pathological hypertrophy (Fig. 5).

Dissimilar to the present study, previous studies planned $\mathrm{GH}$ administration based on the therapeutic approach in children or adults with GH deficiency (4). Various studies have been performed on the use of $\mathrm{r}-\mathrm{hGH}$ as a doping hormone in sports $(5,6)$. However, the present study is seemingly the first to investigate the effects of r-hGH administration in conjunction with swimming exercise on PI3K/AKT/ mTOR and ERK signaling pathways, known to regulate cardiac hypertrophy, and on miRNAs associated with cardiac hypertrophy (miR-21 and miR-133) in rats. In the present study, r-hGH was administered at $0.3 \mathrm{mg} / \mathrm{kg}$ (1 IU/day) concurrently with swimming exercise 5 times a week for 8 weeks.

A limitation of the current study is the evaluation of one dose level, when different doses may demonstrate the potential harmful effects of r-hGH. We plan to assess the effects of higher dose $\mathrm{r}$-hGH treatment in the future.

Heart weight/ body weight, myocyte diameter and sedentary bradycardia are among the determinants of physiological left ventricular hypertrophy $(7,22,28)$. The current study data demonstrated left ventricular hypertrophy formation based on increase in to heart weight/ body weight ratio (HWI) in SE and SE-GH rats. 

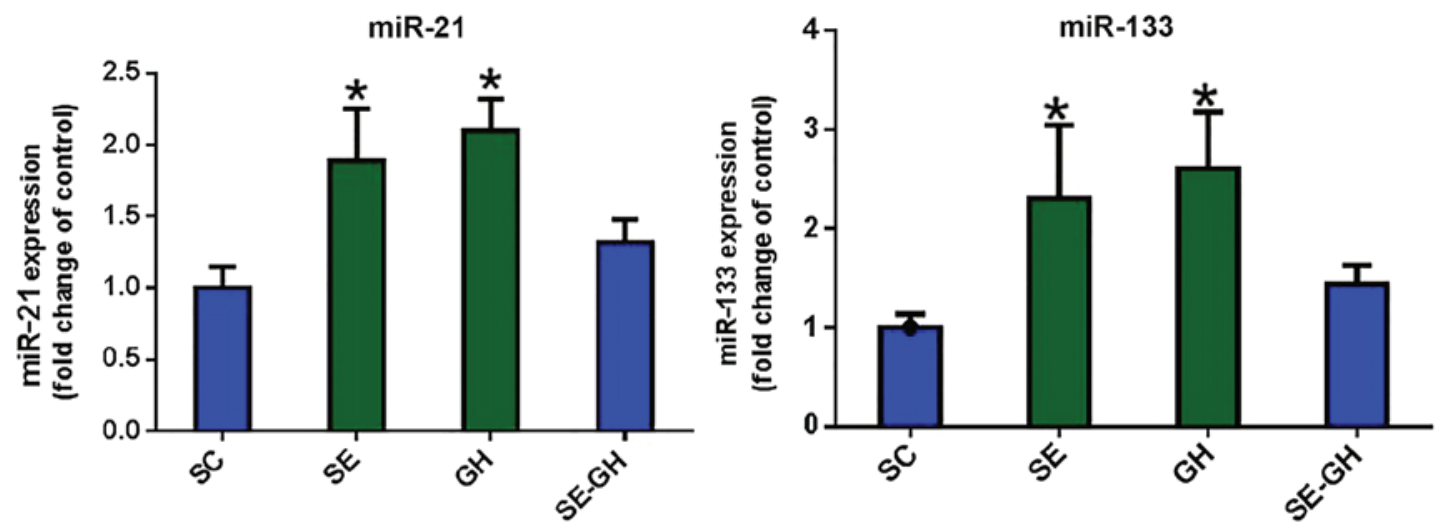

Figure 4. Effect of swimming exercise and/or r-hGH administration on miR-21 and miR-133 expression in left ventricular tissue. ${ }^{*} \mathrm{P}<0.05$. SC, sedentary control; SE, swimming exercise; GH, r-hGH exposure; SE-GH, swimming exercise-r-hGH exposure; r-hGH, recombinant human growth hormone; miR, microRNA.

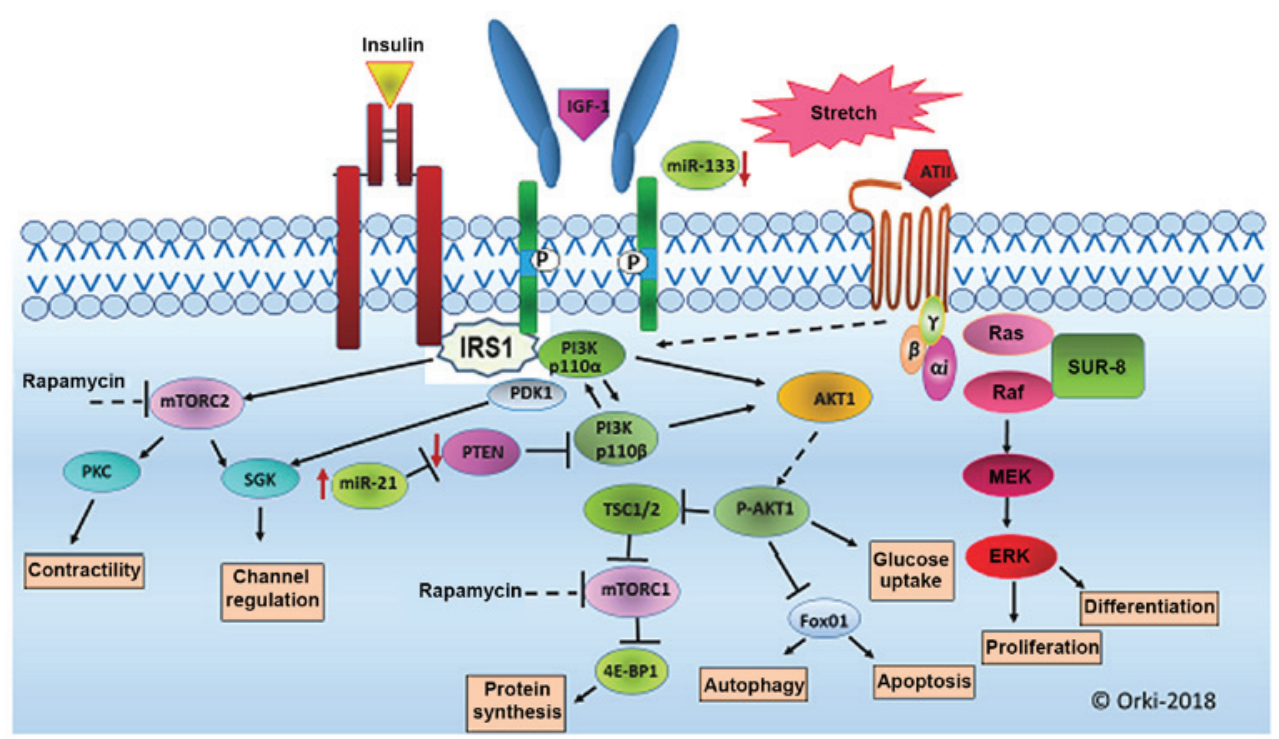

Figure 5. PI3K/AKT/mTOR and ERK signalling pathways associated with cardiac hypertrophy. Signal transduction pathways critical for myosin growth include PI3K/AKT/mTOR, MAPK/ERK. The PI3K/AKT/mTOR signaling pathway has been reported to serve a regulatory role in cell growth, metabolism, survival and angiogenesis, and to govern the development and transformation of left ventricular hypertrophy. The ERK signaling pathway, the members of which belong to the MAPK family, comprises a cascade of a series of successively acting kinases that finally lead to phosphorylation and activation of terminal kinases including p38, c-Jun N-terminal kinases and ERK. miR-133 has a critical role in determining cardiomyocyte hypertrophy and miR-21 has also been implicated in the regulation of cardiac fibrosis and cardiac muscle contractility, and has been shown to negatively regulate phosphatase and tensin homolog (PTEN) gene expression. PI3K, phosphoinositide-3-kinase; AKT, serine/threonine protein kinase; mTOR, mechanistic target of rapamycin; MAPK, mitogen-activated protein kinase; ERK, extracellular signal-regulated kinase; Ang II, angiotensin II; DAG, diacylglycerol; Et, endothelin; FGF, fibroblast growth factor; G, G protein; IGF, insulin-like growth factor; IP3, inositol 1,4,5-triphosphate; MEK, MAP kinase kinase; NFAT, nuclear factor of activated T cells; PKC, protein kinase C; PLC, phospholipase C; RAF, mitogen-activated protein (MAP) kinase kinase kinase; RAS, monomeric GTPase.

ANP, $\beta$-MHC and $\alpha$-actin levels have been reported as molecular markers of pathological hypertrophy $(32,33)$. Increased expression of ANP and $\beta$-MHC was found in the SE-GH group in the current study; while relatively small changes were determined in ANP gene expression. No changes in these factors were identified in the SE and GH groups. It is noteworthy that markedly greater increases in ANP gene expression have been reported under conditions of acute pressure or volume overload (10- to 20 -fold increase), which may represent a different pathogenetic response (29).

Previous studies have shown that activation of the PI3K/AKT signaling cascade results in myocardial hypertrophy $(33,34)$. In a cardiac hypertrophy study utilizing aortic narrowing surgery, PI3K-negative transgenic mice (dnPI3K) were determined to have smaller hearts (appearance and weight) compared with controls. Additionally, a significant increase in heart weight was observed among dNPI $3 \mathrm{~K}$ mice compared with the control group in response to placement of an aortic band and swimming exercise (8). However, it has been suggested that PI3K signaling is not essential for pathological hypertrophy development induced by increased pressure, but is important for physiological cardiac hypertrophy development induced by swimming exercise (34). In the present study, PI3K gene and $\mathrm{PI} 3 \mathrm{~K} \alpha$ protein expression levels were increased to a greater extent in the SE-GH group compared with levels in the other groups. Furthermore, HWI values were significantly higher in the SE-GH group. This increase appeared to be a result of both the swimming exercise and r-hGH administration. This 
suggests that $\mathrm{r}-\mathrm{hGH}$ administration for 8 weeks does not cause as much damage as pathological cardiac hypertrophy induced by an aortic band, but differs from the physiological hypertrophy induced by swimming exercise. Greater understanding of the mechanism involved requires further studies performed with higher doses of r-hGH over longer durations.

PTEN is established as a major negative regulator of the PI3K/AKT signaling pathway (35). Interestingly, heart weight has been reported to be increased (by 50\%) in PTEN (null) rats (36). Certain studies have demonstrated negative regulation of PTEN gene expression by miR-21 (31). miR-21 has also been shown to serve a role in cardiomyocyte hypertrophy (37). In a study by Ma et al (7), PTEN expression was significantly decreased at the gene and protein levels in the SE group compared with in the control group, and the expression of PTEN-targeting miR-21 was also observed to be significantly increased. Similarly, in the present study, a significant decrease in PTEN was observed in the SE group compared with in the control group, concomitant with a significant increase in miR-21 gene expression. However, in the GH and SE-GH groups, no difference was observed in PTEN gene expression compared with the control, while miR-21 was significantly increased in the GH group; the miR-21 expression was similar to that of controls in the SE-GH group. These findings suggests that $\mathrm{r}-\mathrm{hGH}$ administration may impair the regulation between miR-21 and its target PTEN.

AKT-1 has been demonstrated to be necessary for the development of physiological hypertrophy induced by exercise (38) and for the regulation of normal cardiac growth (39). Previous reports have concluded that cardiac AKT-1 may regulate cardiac hypertrophy when studying models of cardiac hypertrophy or ischemia $(32,38)$. In the study of Ma et al (7), it was concluded that AKT1 upregulated PI3K $\alpha$ through phosphorylation in left ventricular hypertrophy induced by swimming exercise. Left ventricular hypertrophy (LVH) induced by swimming exercise training is an important physiological mechanism that compensates for chronic increases in hemodynamics (7). Its comparison to the previously reported Akt2 deficiency phenotype reveals the non-redundant functions of Akt1 and Akt2 genes with respect to organismal growth and insulin-regulated glucose metabolism (39). To assess the role of AKT1, the present study tested AKT1 gene and p-AKT1 protein expression. A significant increase in AKT1 gene expression was observed in the SE-GH group compared with in the controls, and p-AKT1 protein levels were significantly higher in the GH and SE-GH groups than in the control group. This demonstrates that AKT1 may increase the upregulation of $\mathrm{PI} 3 \mathrm{~K} \alpha$ and result in the relatively highest elevation in PI3K gene expression observed in the SE-GH group. Expression of the mTOR gene has been associated with decreased cardiac hypertrophy through inhibition of mTOR with rapamycin in humans and animal models $(34,40)$. In the current study, phospho ${ }^{\text {ser } 2448}$ mTOR protein expression was determined to be increased in SE, GH and SE-GH rats compared with in controls. These results may aid in clarifying the cause of hypertrophy following SE-GH exposure.

It has been reported that the activation of cardiac myocytes in response to any stress stimulation occurs by means of the ERK1/2 signaling pathway (8). Exercise and pressure overload may activate the ERK1/2 signaling pathway $(10,39)$. Another study has suggested that the hypertrophic response to exercise-induced load increase is not reduced, although phosphorylation of ERK1/2 is essentially eliminated, following the stimulation of increased pressure $(40,41)$. Additionally, it has been reported that MAPK kinase-ERK1/2 inhibition does interfere with hypertrophic morphology (19). In the present study, it was observed that post-exercise development of left ventricular hypertrophy did not differ between the groups in terms of ERK1/2 signaling: ERK1/2 gene and protein expression was similar across all groups. With this, the data indicates that the ERK1/2 signaling pathway may not be activated during physiological left ventricular hypertrophy caused by swimming exercise and $\mathrm{r}-\mathrm{hGH}$ administration over 8 weeks.

miR-133, which is established to be highly expressed in adult heart and skeletal muscles, serves a role in cell differentiation and proliferation $(42,43)$. miR-133 has a critical role in determining cardiomyocyte hypertrophy; its overexpression may inhibit hypertrophy whereas its suppression may induces hypertrophy in vitro and in vivo (20). Previous study has shown that miR-133 may controls cardiac hypertrophy and decrease significantly in the presence of cardiac hypertrophy and heart failure (44). Thus, the reduction of miR-133 expression may be involved in the development and progression of chronic heart failure (45). The present study identified increased miR-133 expression in the ventricular tissue of SE and GH rats compared with controls. However, the SE-GH group was similar to the controls with regard to miR-133 expression.

In conclusion, the present study demonstrated that $\mathrm{r}-\mathrm{hGH}$ administration with swimming exercise over 8 weeks altered PI3K, PTEN, AKT-1 and mTOR expression in rat heart. As these factors are involved in the PI3K/AKT/mTOR signaling pathway, as a major regulator of cell proliferation, metabolism, survival and angiogenesis, these changes may have contributed to cardiac hypertrophy. Additionally, r-hGH administration was observed to impair the negative regulation between miR-21 and its target, PTEN. Greater understanding of this mechanism requires further studies on more genes and miRNAs involved in the PI3K/AKT/mTOR and ERK signaling pathways.

\section{Acknowledgments}

Not applicable.

\section{Funding}

The current study was funded by Trakya University Scientific Research Projects Unit (Project no. 2015-36; Edirne, Turkey).

\section{Availability of data and materials}

The materials and data described in the manuscript are available on reasonable request for non-commercial purposes.

\section{Authors' contributions}

OP designed the experiments, and coordinated the project and interpreted results. SAV interpreted the results. ZBD performed molecular studies and interpreted results. ET was responsible for morphological evaluations and interpretation of results. PT and AD were involved in the animal protocols and determination of blood parameters. 


\section{Ethics approval and consent to participate}

The study was conducted in the Trakya University Local Experimental Animals Research Center after obtaining approval from the Local Experimental Animals Ethics Committee. (approval no. TUHADYEK:2015/10).

\section{Patient consent for publication}

Not applicable.

\section{Competing interests}

The authors declare that they have no competing interests.

\section{References}

1. Graham MR, Baker JS, Evans P, Kicman A, Cowan D, Hullin D, Thomas N and Davies B: Physical effects of short-term recombinant human growth hormone administration in abstinent steroid dependency. Horm Res 69: 343-354, 2008.

2. Jenkins PJ: Growth hormone and exercise. Clin Endocrinol (Oxf) 50: 683-689, 1999.

3. Castellano G, Affuso F, Conza PD and Fazio S: The GH/IGF-1 Axis and Heart Failure. Curr Cardiol Rev 5: 203-215, 2009.

4. Colao A, Marzullo P, Di Somma C and Lombardi G: Growth hormone and the heart. Clin Endocrinol (Oxf) 54: 137-154, 2001.

5. WorldAnti-Doping Agency:The2010ProhibitedList.International Standard. https://www.wada-ama.org/sites/default/files/ resources/files/WADA_Prohibited_List_2010_EN.pdf.Accessed September 19, 2009.

6. Voss SC, Giraud S, Alsayrafi M, Bourdon PC, Schumacher YO, Saugy M and Robinson N: The effect of a period of intensive exercise on the isoform test to detect growth hormone doping in sports. Growth Horm IGF Res 23: 105-108, 2013.

7. Ma Z, Qi J, Meng S, Wen B and Zhang J: Swimming exercise training-induced left ventricular hypertrophy involves microRNAs and synergistic regulation of the PI3K/AKT/mTOR signaling pathway. Eur J Appl Physiol 113: 2473-2486, 2013.

8. Heineke J and Molkentin JD: Regulation of cardiac hypertrophy by intracellular signalling pathways. Nat Rev Mol Cell Biol 7: 589-600, 2006

9. Guertin DA and Sabatini DM: Defining the role of mTOR in cancer. Cancer Cell 12: 9-22, 2007.

10. Sugden PH and Clerk A: 'Stress-responsive' mitogen-activated protein kinases (c-Jun N-terminal kinases and p38 mitogen-activated protein kinases) in the myocardium. Circ Res 83: 345-352, 1998.

11. Purcell NH, Wilkins BJ, York A, Saba-El-Leil MK, Meloche S Robbins J and Molkentin JD: Genetic inhibition of cardiac ERK1/2 promotes stress-induced apoptosis and heart failure but has no effect on hypertrophy in vivo. Proc Natl Acad Sci USA 104: 14074-14079, 2007.

12. Hutvágner G and Zamore PD: A microRNA in a multiple-turnover RNAi enzyme complex. Science 297: 2056-2060, 2002.

13. Bartel DP: MicroRNAs: genomics, biogenesis, mechanism, and function. Cell 116: 281-297, 2004.

14. Bao H, Hu S, Zhang C, Shi S, Qin W, Zeng C, Zen K and Liu Z: Inhibition of miRNA-21 prevents fibrogenic activation in podocytes and tubular cells in Ig A nephropathy. Biochem Biophys Res Commun 444: 455-460, 2014.

15. Da Costa Martins PA and De Windt LJ: Targeting microRNA targets. Circ Res 111: 506-508, 2012.

16. Cheng YS, Tang YQ, Dai DZ and Dai Y: AQP4 knockout mice manifest abnormal expressions of calcium handling proteins possibly due to exacerbating pro-inflammatory factors in the heart. Biochem Pharmacol 83: 97-105, 2012.

17. Da Costa Martins PA and De Windt LJ: MicroRNAs in control of cardiac hypertrophy. Cardiovasc Res 93: 563-572, 2012

18. Wang L, Li X, Zhou Y, Shi H, Xu C, He H, Wang S, Xiong X, Zhang Y, Du Z, et al: Downregulation of miR-133 via MAPK/ERK signaling pathway involved in nicotine-induced cardiomyocyte apoptosis. Naunyn Schmiedebergs Arch Pharmacol 387: 197-206, 2014
19. Dong DL, Chen C, Huo R, Wang N, Li Z, Tu YJ, Hu JT, Chu X, Huang W and Yang BF: Reciprocal repression between microRNA-133 and calcineurin regulates cardiac hypertrophy: A novel mechanism for progressive cardiac hypertrophy. Hypertension 55: 946-952, 2010.

20. Dong S, Ma W, Hao B, Hu F, Yan L, Yan X, Wang Y, Chen Z and Wang Z: microRNA-21 promotes cardiac fibrosis and development of heart failure with preserved left ventricular ejection fraction by up-regulating Bcl-2. Int J Clin Exp Pathol 7: 565-574, 2014.

21. DA Silva ND Jr, Fernandes T, Soci UP, Monteiro AW, Phillips MI and DE Oliveira EM: Swimming training in rats increases cardiac MicroRNA-126 expression and angiogenesis. Med Sci Sports Exerc 44: 1453-1462, 2012.

22. Oliveira EM, Sasaki MS, Cerêncio M, Baraúna VG and Krieger JE: Local renin-angiotensin system regulates left ventricular hypertrophy induced by swimming training independent of circulating renin: A pharmacological study. J Renin Angiotensin Aldosterone Syst 10: 15-23, 2009.

23. Livak KJ and Schmittgen TD: Analysis of relative gene expression data using real-time quantitative PCR and the 2(-Delta Delta C(T)) method. Methods 25: 402-408, 2001.

24. Hirata Y, Murai N, Yanaihara N, Saito M, Saito M, Urashima M, Murakami Y, Matsufuji S and Okamoto A: MicroRNA-21 is a candidate driver gene for 17q23-25 amplification in ovarian clear cell carcinoma. BMC Cancer 14: 799, 2014.

25. Iijima Y, Seike M, Noro R, Ibi T, Takeuchi S, Mikami I, Koizumi K, Usuda J and Gemma A: Prognostic significance of PIK3CA and SOX2 in Asian patients with lung squamous cell carcinoma. Int J Oncol 46: 505-512, 2015.

26. Zhou X, Tan M, Stone Haw thorne V, Klos KS, Lan KH, Yang Y, Yang W, Smith TL, Shi D and Yu D: Activation of the $\mathrm{Akt} / \mathrm{mammalian}$ target of rapamycin/4E-BP1 pathway by ErbB2 overexpression predicts tumor progression in breast cancers. Clin Cancer Res 10: 6779-6788, 2004.

27. Domenighetti AA, Danes VR, Curl CL, Favaloro JM, Proietto J and Delbridge LM: Targeted GLUT-4 deficiency in the heart induces cardiomyocyte hypertrophy and impaired contractility linked with $\mathrm{Ca}(2+)$ and proton flux dysregulation. J Mol Cell Cardiol 48: 663-672, 2010.

28. Chung E and Diffee GM: Effect of aging on power output properties in rat skinned cardiac myocytes. J Gerontol A Biol Sci Med Sci 66: 1267-1273, 2011

29. Vikstrom KL, Bohlmeyer T, Factor SM and Leinwand LA Hypertrophy, pathology, and molecular markers of cardiac pathogenesis. Circ Res 82: 773-778, 1998.

30. Saugy M, Robinson N, Saudan C, Baume N, Avois L and Mangin P: Human growth hormone doping in sport. Br J Sports Med 40 (Suppl 1): i35-i39, 2006.

31. Cheng Y, Ji R, Yue J, Yang J, Liu X, Chen H, Dean DB and Zhang C: MicroRNAs are aberrantly expressed in hypertrophic heart: Do they play a role in cardiac hypertrophy? Am J Pathol 170: 1831-1840, 2007.

32. Soci UP, Fernandes T, Hashimoto NY, Mota GF, Amadeu MA, Rosa KT, Irigoyen MC, Phillips MI and Oliveira EM: MicroRNAs 29 are involved in the improvement of ventricular compliance promoted by aerobic exercise training in rats. Physiol Genomics 43: 665-673, 2011.

33. McMullen JR and Jennings GL: Differences between pathological and physiological cardiac hypertrophy: Novel therapeutic strategies to treat heart failure. Clin Exp Pharmacol Physiol 34: 255-262, 2007.

34. McMullen JR, Shioi T, Zhang L, Tarnavski O, Sherwood MC, Kang PM and Izumo S: Phosphoinositide 3-kinase(p110alpha) plays a critical role for the induction of physiological, but not pathological, cardiac hypertrophy. Proc Natl Acad Sci USA 100: 12355-12360, 2003

35. Maehama T and Dixon JE: PTEN: A tumour suppressor that functions as a phospholipid phosphatase. Trends Cell Biol 9: 125-128, 1999

36. Crackower MA, Oudit GY, Kozieradzki I, Sarao R, Sun H, Sasaki T, Hirsch E, Suzuki A, Shioi T, Irie-Sasaki J, et al: Regulation of myocardial contractility and cell size by distinct PI3K-PTEN signaling pathways. Cell 110: 737-749, 2002.

37. Bai L, Liang R, Yang Y, Hou X, Wang Z, Zhu S, Wang C, Tang Z and Li K: MicroRNA-21 Regulates PI3K/Akt/mTOR Signaling by Targeting TGF $\beta$ I during Skeletal Muscle Development in Pigs. PLoS One 10: e0119396, 2015.

38. DeBosch B, Treskov I, Lupu TS, Weinheimer C, Kovacs A, Courtois $\mathrm{M}$ and Muslin AJ: Akt1 is required for physiological cardiac growth. Circulation 113: 2097-2104, 2006. 
39. Cho H, Thorvaldsen JL, Chu Q, Feng F and Birnbaum MJ: Akt1/PKBalpha is required for normal growth but dispensable for maintenance of glucose homeostasis in mice. J Biol Chem 276 : 38349-38352, 2001.

40. Shioi T, McMullen JR, Tarnavski O, Converso K, Sherwood MC, Manning WJ and Izumo S: Rapamycin attenuates load-induced cardiac hypertrophy in mice. Circulation 107: 1664-1670, 2003.

41. Leontieva OV, Paszkiewicz GM and Blagosklonny MV: Mechanistic or mammalian target of rapamycin (mTOR) may determine robustness in young male mice at the cost of accelerated aging. Aging (Albany NY) 4: 899-916, 2012.

42. Chen JF, Mandel EM, Thomson JM, Wu Q, Callis TE, Hammond SM, Conlon FL and Wang DZ: The role of microRNA-1 and microRNA-133 in skeletal muscle proliferation and differentiation. Nat Genet 38: 228-233, 2006.
43. Horie T, Ono K, Nishi H, Iwanaga Y, Nagao K, Kinoshita M, Kuwabara Y, Takanabe R, Hasegawa K, Kita $\mathrm{T}$, et al: MicroRNA-133 regulates the expression of GLUT4 by targeting KLF15 and is involved in metabolic control in cardiac myocytes. Biochem Biophys Res Commun 389: 315-320, 2009.

44. van Rooij E, Sutherland LB, Liu N, Williams AH, McAnally J, Gerard RD, Richardson JA and Olson EN: A signature pattern of stress-responsive microRNAs that can evoke cardiac hypertrophy and heart failure. Proc Natl Acad Sci USA 103: 18255-18260, 2006.

45. Abdellatif M: The role of microRNA-133 in cardiac hypertrophy uncovered. Circ Res 106: 16-18, 2010.

(i) 19 This work is licensed under a Creative Commons Attribution-NonCommercial-NoDerivatives 4.0 International (CC BY-NC-ND 4.0) License. 